\title{
Surveillance of Patulin in Apple, Grapes, Juices and Value-Added Products for Sale in Pakistan
}

\author{
Shabbir Hussain 1,2, Muhammad Rafique Asi 1,* ${ }^{1,}$ Mazhar Iqbal ${ }^{3}{ }^{(1)}$, Muhammad Akhtar ${ }^{4}$, \\ Muhammad Imran ${ }^{4}$ and Agustín Ariño ${ }^{5, * \text { D }}$ \\ 1 Food Toxicology Laboratory, Nuclear Institute for Agriculture and Biology College (NIAB-C), \\ Pakistan Institute of Engineering and Applied Sciences (PIEAS), Jhang Road, Faisalabad 38000, Pakistan; \\ shabbir.ne@gmail.com \\ 2 Central Analytical Facility Division (CAFD), Pakistan Institute of Nuclear Science and \\ Technology (PINSTECH), P. O. Nilore, Islamabad 45650, Pakistan \\ 3 Health Biotechnology Division, National Institute for Biotechnology and Genetic Engineering \\ College (NIBGE-C), Pakistan Institute of Engineering and Applied Sciences (PIEAS), Jhang Road, \\ Faisalabad 38000, Pakistan; hamzamgondal@gmail.com \\ 4 Soil \& Environmental Sciences Division, Nuclear Institute for Agriculture and Biology College (NIAB-C), \\ Jhang Road, Faisalabad 38000, Pakistan; akhtarniab@gmail.com (M.A.); imran1631@gmail.com (M.I.) \\ 5 Instituto Agroalimentario de Aragón-IA2 (Universidad de Zaragoza-CITA), Facultad de Veterinaria, \\ 50013 Zaragoza, Spain \\ * Correspondence: asimuhammad@yahoo.co.uk (M.R.A.); aarino@unizar.es (A.A.)
}

Received: 7 October 2020; Accepted: 24 November 2020; Published: 26 November 2020

\begin{abstract}
The prime objective of the current study was to investigate the occurrence of mycotoxin patulin (PAT) in apples, grapes and their value added products. PAT was determined by a validated method based on HPLC with UV detector. A total of 381 samples comprising apple and grape fruits ( $n=133$ each), apple-based products ( $n=76$, juice, puree, jam) and grape juice $(n=39)$ were analyzed. PAT was found in $58.9 \%$ samples of apple and apple-based products, with a mean of $49.8 \mu \mathrm{g} / \mathrm{kg}$ (maximum $396 \mu \mathrm{g} / \mathrm{kg}$ ), while 27.3\% samples contained PAT beyond the maximum regulatory limit of $50 \mu \mathrm{g} / \mathrm{kg}$. The average levels of PAT contamination in apple-derived products was higher in apple juice concentrate, followed by apple puree, apple juice and apple jam. The incidence of PAT in table grapes was $65.1 \%$, with a mean of $53.9 \mu \mathrm{g} / \mathrm{kg}$ (maximum $505 \mu \mathrm{g} / \mathrm{kg}$ ), whereas $23.8 \%$ exceeded the maximum level. Among the fruit samples, there were differences in PAT contents due to apple variety (6 types) or grape variety (8 types), as well as for sampling location. Our investigations showed the wide PAT occurrence in fruits and derived value-added products affecting consumer product safety, so that the population is chronically exposed to this toxin.
\end{abstract}

Keywords: patulin; apple; grapes; fruit products; occurrence; risk

\section{Introduction}

Pakistan is located in South Asia with an extremely diverse climate that varies from tropical to temperate and even cold in the northern part. The diversity of the landscape and climate in Pakistan allows a wide variety of fruit trees that produce fruits with good flavor and taste, which are available throughout the year. Apple fruits are cultivated and harvested in the northern geographic area whereas grapes are mainly grown in the central part of the country. The different fruit varieties are cultivated in an area of around 800,000 hectares (11\% Ha under apples and $2 \%$ Ha for grapes) with a total production of 7.05 million tons (8\% apples and 1\% grapes) [1]. During the 2017-2018 crop season, only $10 \%$ of the entire fruit production was exported. Fruits are generally high in fiber, vitamin $\mathrm{C}$, beta-carotene and antioxidants, and have a significant role in the human diet, as their intake 
is associated with a reduced risk of cardiac disease, all-cause mortality and cancer [2]. Apples and grapes are eaten fresh and are preserved as jams, as well as processed as fruit juices or used as ingredients in many manufactured foods.

Many fruits are perishable and susceptible to fungal attack that cause maceration and decay that reduces quality, and can lead to the synthesis of mycotoxins like patulin (PAT). This mycotoxin is frequently linked with fruits, their juices and value added products comprising foods for children, and it is a common contaminant of apple and apple-based products [3]. Patulin mycotoxin is primarily produced by toxigenic strains of the fungi Penicillium expansum, Aspergillus clavatus and Byssochlamys nivea, though the blue-mold disease, triggered by P. expansum, is the utmost postharvest disease of fruits in storage [4]. This fungus can infect fruits before or during harvest via injuries, with the subsequent patulin production during post-harvest [5]. Conventional techniques for PAT testing have used multi-step liquid-liquid extraction (LLE), solid-phase extraction (SPE) or molecularly imprinted polymers (MIP), followed by determination by liquid chromatography with ultraviolet, diode array or mass spectrometry detection [6].

There are numerous aspects that affect patulin incidence and its contamination in fruits, such as climate conditions, type and cultivar of fruits, year of production, geographical location, pre- and post-harvest managements, storage environments and surface damage on the fruits [7]. Snini et al. [8] showed that PAT is a cultivar-dependent factor supporting the colonization of apple fruits by P. expansum. Among thirteen different apple varieties, the majority showed significant differences in the progress of fungal decay and the total quantities of PAT. The cultivar influence for patulin in grapes has not been previously identified although it has been extensively studied in wine grapes for other mycotoxins such as ochratoxin A [9].

Patulin has been stated to cause gastrointestinal disorders with ulceration and bleeding, as well as genotoxicity, neurotoxicity, hepatotoxicity and immunotoxicity [10]. Because of the toxicological implications of PAT, several regulatory institutions worldwide have established maximum levels of patulin, mainly in the apple-based products, ranging from 25 to $50 \mu \mathrm{g} / \mathrm{kg}$. The Codex Alimentarius [11] set the maximum permissible PAT levels in fruits and their juices at $50 \mu \mathrm{g} / \mathrm{kg}$. According to the Commission Regulation (EC) No. 1881/2006, the European Union (EU) fixed limits of patulin in fruit juices $(50 \mu \mathrm{g} / \mathrm{kg})$, solid apple products $(25 \mu \mathrm{g} / \mathrm{kg})$ and foods for infants and young children $(10 \mu \mathrm{g} / \mathrm{kg})[12]$.

In view of the above facts, the focus of the current research was to assess the incidence of patulin in apple and grape fruits, along with their value added products, collected during two years in Pakistan, and compare the levels with the regulatory maximum limits. In addition, the outcomes of the present survey will also allow decisions on control strategies to be taken derived from objective data.

\section{Materials and Methods}

\subsection{Sampling}

Random samples of fresh fruits, juices and derived products (purees and jams) for sale were procured from city markets, supermarkets and general stores from different areas of Pakistan during the years 2018 and 2019. A total of 133 samples of fresh apple fruits designated for direct consumption were taken, out of which 70 from different varieties (Amri 9, Kashmiri 11, Kala Kulu 15, Golden delicious 14, Red delicious 13, and Gaja 8) and 63 samples from different cities (Rawalpindi 10, Faisalabad 15, Lahore 8, Gilgit 7, Swat 8, Muzafarabad 9 and Murree 6). For the study of the different apple varieties, the samples were collected from orchard owners and from fruit and vegetable wholesale market in Faisalabad. In Pakistan, the apple varieties are mostly cultivated in Baluchistan and Khyber Pakhtunkhwa provinces of Pakistan, as well as in Azad Jammu and Kashmir. The fruits from these areas are transported throughout the country, especially to major cities. For the study of the different sampling locations, the apple samples were directly purchased from vendors and fruit shops located in the vicinity of the cities, but the variety was not specifically recorded because the sellers did not have such information. 
Additionally, 76 samples of apple-derived products (juice 35, concentrate 10, puree 21, jam 10) belonging to commercial brands of nation-wide distribution were taken. Similarly, a total of 133 samples of fresh table grapes were sampled, out of which there were 79 from different varieties (White Thomson 6, King ruby 9, Narc black 12, Perlette 8, Sundar Khani 14, Vitro black 10, Cardinal 9, Flame 11) and 54 samples from different cities (Faisalabad 16, Lahore 10, Rawalpindi 10, Attock 7, Dunyapur 5 and Chakwal 6). For the study of the different grapes varieties, the samples were collected from orchard owners and from a fruit and vegetable wholesale market in Faisalabad. In Pakistan, the table grape varieties are mainly cultivated in central Punjab, Baluchistan and Khyber Pakhtunkhwa provinces of Pakistan, and transported to other regions of the country. For the study of the different sampling locations, the grape samples were procured from vendors and fruit shops located in the vicinity of the cities, but the sellers did not provide varietal information. In addition, 39 samples of grape juice from different brands were taken. Fresh fruits (1 kg), fruit juices (1 L) and derived products (500 g package) were stockpiled at $4{ }^{\circ} \mathrm{C}$ in their original packages in Food Toxicology Laboratory at NIAB Faisalabad until analysis. All the stored samples were opened and homogenized thoroughly before analysis.

\subsection{Chemicals and Reagents}

HPLC grade glacial acetic acid, acetonitrile, and ethyl acetate, and analytical-grade sodium chloride, sodium sulfate anhydrous and sodium carbonate were purchased from Merck (Darmstadt, Germany). Patulin standard, 5-hydroxymethyl furfural (5-HMF) and pectinase enzyme solution were supplied by Sigma-Aldrich (Saint-Louis, MO, USA). Stock reference standard solution of PAT $1.0 \mathrm{mg} / \mathrm{mL}$ was prepared in HPLC-grade acetonitrile and stockpiled at $-4{ }^{\circ} \mathrm{C}$. Required volumes of working standard solutions $(5,10,30,50,70$, and $100 \mu \mathrm{g} / \mathrm{L})$ were prepared from a stock solution in $0.1 \%$ acetic acid. Multifunctional cleanup columns PriboFast ${ }^{\circledR}$, MFC-228 were obtained from Pribolab (Singapore).

\subsection{Samples Preparation, Extraction and Cleanup}

The procured samples of fruits were selected without debris, washed adequately with tape and distilled water, dried in the shade and cut into small slices using a sharp knife. Around $500 \mathrm{~g}$ sample was thoroughly homogenized by a high-speed mixer blender (Mix 2000, Braun Blender, Marktheidenfel, Germany). All samples of fruit products (juice, jam and puree) were directly taken from original stored packages. The analytical technique for PAT assay was established based on AOAC, method 2000.02 [13]. For fresh fruits and clear juices, no preparation was required. For cloudy juices, such as puree and jam, a sample portion $(25 \mathrm{~mL}$ or $25 \mathrm{~g})$ was pretreated with $150 \mu \mathrm{L}$ pectinase enzyme and incubated for $2 \mathrm{~h}$ at $40{ }^{\circ} \mathrm{C}$ to clarify the sample prior to extraction. Homogenized fruit and product samples ( $25 \mathrm{~mL}$ or $25 \mathrm{~g}$ ), were extracted two times using $50 \mathrm{~mL}$ ethyl acetate accompanied by $2 \mathrm{~g}$ sodium chloride in the $250 \mathrm{~mL}$ Erlenmeyer flask. The contents of flask were shaken using a horizontal shaker (Gunther, and $\mathrm{Co}$, Bremen, Germany) at a high speed for about one hour. The organic upper layers (combined) were shaken with $10 \mathrm{~mL}$ of sodium carbonate $\left(1.5 \%, \mathrm{Na}_{2} \mathrm{CO}_{3}\right.$ solution). The lower aqueous layer was drained and the top layer was quickly dehydrated using $15 \mathrm{~g}$ of anhydrous powder sodium sulfate $\left(\mathrm{Na}_{2} \mathrm{SO}_{4}\right)$, and then filtered by using filter paper (Whatman No. 1). The filtrate was subjected to clean-up through MFC 228 multifunctional column, and purified extract $4 \mathrm{~mL}$ was collected in liquid scintillation vial. The extracts of samples were evaporated under stream of nitrogen to dryness. The dried residue was instantly dissolved in $1 \mathrm{~mL}$ of $0.1 \%$ acetic acid, and filtered through a $0.22 \mu \mathrm{m}$ syringe filter (Millipore, Darmstadt, Germany) to get a clear solution. The extracted samples were then analyzed for the PAT assay by reverse phase HPLC system equipped with ultraviolet-Vis detector (SPD 10AS, Shimadzu, Japan) using an isocratic mode of operation. The total run time was set at $10 \mathrm{~min}$ and $20 \mu \mathrm{L}$ volume of sample was injected for PAT analysis.

\subsection{Apparatus and Conditions of HPLC for Analysis}

The apparatus used for the present study was HPLC (LC-10A Shimadzu, Kyoto, Japan) equipped with UV-Visible detector (SPD-10AS) at a wave length of $276 \mathrm{~nm}$. Separations were performed using 
Discovery HS C-18 silica-based column $(250 \times 4.6 \mathrm{~mm}$, particle size $5 \mu \mathrm{m}$; Supelco, Bellefonte, PA, USA), temperature sustained at $30^{\circ} \mathrm{C}$ with $1.5 \mathrm{~mL} / \mathrm{min}$ flow rate in isocratic mode. The mobile phase used was a mixture of acetonitrile and water $(10: 90, v / v)$. A Rheodyne injector $(20 \mu \mathrm{L}$ loop) was used for injection of standards and sample extracts, and Software CLASS LC-10 (version 1.63, Shimadzu, Kyoto, Japan) was used for acquisition of chromatograms. Patulin was confirmed by its retention time ( $\approx 6.558 \mathrm{~min})$ according to a reference standard, and quantified by measuring the peak area according to a standard curve. For validation method, a simple test was executed to confirm the separation of PAT peak and 5 -HMF peak that is its principal interference.

\subsection{Statistical Analysis}

Statistical analysis was executed for the compiled data. The Excel software was used to calculate descriptive statistics of patulin in fruits, their juices and their derived products and data were presented as a mean along with standard deviation. The samples with levels of PAT greater than the limit of detection (LOD) were considered as positive. The mean was calculated considering zero value, for the samples with PAT levels less than LOD. The significance differences in patulin concentrations between the groups were investigated using a one-way ANOVA $(p<0.05)$ test, by SPSS (IBM, SPSS Statistics, 19, Armonk, NY, USA, 2010).

\section{Results and Discussion}

\subsection{Method Validation}

The HPLC-based analytical method was evaluated in terms of linearity, recovery, precision and sensitivity. Linearity was tested by injection of patulin standards at concentrations in the range of 5 to $100 \mu \mathrm{g} / \mathrm{L}$, obtaining a correlation coefficient of 0.9921 . Recovery experiments were operated on spiked samples at different PAT levels $(10,20,50,70$ and $100 \mu \mathrm{g} / \mathrm{kg})$. The mean recoveries of PAT in spiked apples $(n=3)$ were $96.5,94.3,95.1,100.5$ and $101.1 \%$, respectively (mean value $97.5 \%$ ), while recoveries for spiked grapes attained 92.8, 94.0, 93.2, 100.0 and 96.0\%, respectively (mean value 95.2\%). Intra-day $(n=3)$ and inter-day (3 different days) variation values ranged between 4.3 and $7.2 \%\left(\mathrm{RSD}_{\mathrm{r}}\right)$ and from 4.7 to $7.4 \%\left(\mathrm{RSD}_{\mathrm{R}}\right)$, respectively, indicating good precision. The detection limit (LOD) and the limit of quantification (LOQ) values were calculated according to $\mathrm{s} / n=3$ and $\mathrm{s} / n=10$, respectively. The LODs and the LOQs of PAT were $5 \mu \mathrm{g} / \mathrm{kg}$ and $15 \mu \mathrm{g} / \mathrm{kg}$, respectively. The performance criteria for patulin set out in Commission Regulation (EC) No. 401/2006 [14] establishes three set of values, (1) Level < $20 \mu \mathrm{g} / \mathrm{kg}$ : recovery 50 to $120 \%, \mathrm{RSDr} \leq 30, \mathrm{RSDR} \leq 40$, (2) Level $20-50 \mu \mathrm{g} / \mathrm{kg}$ : recovery 70 to $105 \%$, RSDr $\leq 20$, RSDR $\leq 30$, and (3) Level $>50 \mu \mathrm{g} / \mathrm{kg}$ : recovery 75 to $105 \%$, RSDr $\leq 15, \mathrm{RSDR} \leq 25$. Conclusively, the used analytical method fulfilled the performance criteria of Commission Regulation (EC) No. 401/2006 [14] for the accurate determination of patulin levels in foodstuffs.

\subsection{Occurrence of Patulin in Apple Fruits and Their Products}

Incidence and contamination level of PAT in apple fruits and apple-based products are given in Table 1 . The results revealed that 123 out of 209 samples (58.9\%) showed positive for PAT. The positivity in fresh apple fruits was $56.4 \%$, while apple-derived products attained $63.2 \%$. From the six apple fruit varieties analyzed, the prevalence varied from $26.7 \%$ (Kala Kulu) to 100\% (Gaja), while average PAT concentrations were between $32.3 \mu \mathrm{g} / \mathrm{kg}$ (Kala Kulu) and $107.3 \mu \mathrm{g} / \mathrm{kg}$ (Gaja). However, the differences between apple varieties were not significant (ANOVA $p=0.4443$ ). In turn, there were significant differences in PAT levels depending on sampling location (ANOVA $p=0.0162$ ), ascribed to high levels in samples from Murree. The apples coming from this mountain city in the northeast of the country, showed a PAT incidence of $100 \%$ with average levels of $183.8 \mu \mathrm{g} / \mathrm{kg}$, while those from other locations showed incidence from 25 to $70 \%$ and lower levels between 9.4 to $59.2 \mu \mathrm{g} / \mathrm{kg}$. 
Table 1. Occurrence of patulin $(\mu \mathrm{g} / \mathrm{kg})$ in apple fruits and their derived products.

\begin{tabular}{ccccccc}
\hline Sample Type & Variety/Location & $\begin{array}{c}n \text { Total } \\
\text { (Positive) }\end{array}$ & $\begin{array}{c}\text { Incidence } \\
\%\end{array}$ & Mean \pm SD & Max. & $\begin{array}{c}n(\mathbf{\%})> \\
\mathbf{5 0} \boldsymbol{\mu g} / \mathbf{k g}\end{array}$ \\
\hline Apple fruit & Amri & $9(5)$ & 55.6 & $66.9 \pm 94.7$ & 221 & $3(33.3)$ \\
Apple fruit & Kashmiri & $11(7)$ & 63.6 & $100.6 \pm 111.3$ & 264 & $5(45.5)$ \\
Apple fruit & Kala Kulu & $15(4)$ & 26.7 & $32.3 \pm 82.2$ & 308 & $3(20)$ \\
Apple fruit & Golden delicious & $14(9)$ & 64.3 & $68.1 \pm 74.1$ & 189 & $6(42.9)$ \\
Apple fruit & Red delicious & $13(6)$ & 46.2 & $67.6 \pm 103.4$ & 276 & $5(38.5)$ \\
Apple fruit & Gaja & $8(8)$ & 100.0 & $107.3 \pm 106.1$ & 299 & $4(50)$ \\
Apple fruit & Rawalpindi City & $10(7)$ & 70.0 & $55.3 \pm 68.4$ & 182 & $4(40)$ \\
Apple fruit & Faisalabad City & $15(10)$ & 66.7 & $52.2 \pm 75.7$ & 283 & $6(40)$ \\
Apple fruit & Lahore City & $8(4)$ & 50.0 & $57.9 \pm 102.3$ & 277 & $2(25)$ \\
Apple fruit & Gilgit City & $7(3)$ & 42.9 & $16.9 \pm 35.6$ & 97 & $1(14.3)$ \\
Apple fruit & Swat City & $8(2)$ & 25.0 & $9.4 \pm 24$ & 68 & $1(12.5)$ \\
Apple fruit & Muzafarabad City & $9(4)$ & 44.4 & $59.2 \pm 110.7$ & 311 & $2(22.2)$ \\
Apple fruit & Murree City & $6(6)$ & 100.0 & $183.8 \pm 144.2$ & 396 & $5(83.3)$ \\
Apple juice & General stores & $35(22)$ & 62.9 & $5.6 \pm 5.1$ & 18 & $0(0)$ \\
Apple juice concentrate & Supermarket & $10(8)$ & 80.0 & $107.5 \pm 115.5$ & 328 & $5(50)$ \\
Apple puree & City market & $21(15)$ & 71.4 & $29.4 \pm 32.3$ & 99 & $5(23.8)$ \\
Apple jam & General stores & $10(3)$ & 30.0 & $1.7 \pm 2.7$ & 6 & $0(0)$ \\
\hline Total & - & $209(123)$ & 58.9 & $49.8 \pm 83.6$ & 396 & $57(27.3)$ \\
\hline
\end{tabular}

PAT was also examined in samples of apple-based products, the incidence of which ranged from $30 \%$ in apple jam to $80 \%$ in apple juice concentrate. Similarly, the levels were much higher in apple juice concentrate $(107.5 \mu \mathrm{g} / \mathrm{kg})$ than in other products that ranged from 1.7 to $29.4 \mu \mathrm{g} / \mathrm{kg}$. The maximum level of PAT was found in an apple fruit from Murree $(396 \mu \mathrm{g} / \mathrm{kg})$, followed by a sample of apple juice concentrate that amounted to $328 \mu \mathrm{g} / \mathrm{kg}$. Overall, 57 samples of apple fruits and derived products $(27.3 \%)$ surpassed the maximum regulatory limit of $50 \mu \mathrm{g} / \mathrm{kg}$.

Patulin is a toxic substance produced by molds that may grow on apples. The patulin quantity detected in apple products is generally taken as a measured value for the quality of the apples used for the food production. In several studies worldwide, patulin has been found to occur at high levels in apple fruits and derived products offered for sale (Table 2). In a previous survey carried out in Pakistan, high levels of PAT in apple fruits and apple juices were reported by Iqbal et al. [15] PAT was found in apple fruits (36 samples, $66.7 \%$ positive) at $259 \mu \mathrm{g} / \mathrm{kg}$ average level, while mean level in apple juice (29 samples, $51.7 \%$ positive) was $26 \mu \mathrm{g} / \mathrm{kg}$, which is in accordance with the present findings. In an extensive survey carried out in Argentina, PAT showed positive in 40.3\% of 4634 samples of apple fruits, with an average level of $26 \mu \mathrm{g} / \mathrm{kg}$ and a maximum value of $19,622 \mu \mathrm{g} / \mathrm{kg}$ [16]. Vaclavikova et al. [17] carried out a survey of patulin in fresh fruits and derived products for sale in the Czech Republic. In fresh apples and apple pulp, PAT levels varied from 1.3 to $415.2 \mu \mathrm{g} / \mathrm{kg}$, while in apple juice they were from 3.8 and $28.4 \mu \mathrm{g} / \mathrm{kg}$. A study conducted by Al-Hazmi in Saudi Arabia [18] reported patulin in apple juice in the range LOD-152.5 $\mu \mathrm{g} / \mathrm{kg}$. In all mentioned studies, researchers have found the toxin in concentrations beyond the permitted levels. All these findings are well supportive to our results of concentration of PAT in apple fruit and apple-based products.

The high incidence of patulin in apple samples is in line with the survey for postharvest diseases of apples conducted in Punjab (Pakistan), which revealed an occurrence of blue mold caused by Penicillium expansum, which was found with the highest prevalence (56\%) [19]. Patulin contamination is most common on spoiled fruits, often intended for juices and preserves. Its occurrence has been a major issue in the processing of apple juices, as due to its thermal stability, patulin is not destroyed during pasteurization [6]. However, physical adsorption of PAT by the yeast Saccharomyces cerevisiae during the process of fermentation has been reported [20].

Patulin is a mycotoxin produced by fungi belonging to several genera, including Penicillium, Aspergillus and Byssochlamys species. Although patulin can occur in many moldy fruits, grains and other foods, the major sources of patulin contamination are apples and apple products. The presence of molds and mycotoxins play an important role in the depreciation of quality and safety of apples and their derived products. Toxigenic fungi infect fruits either prior to or after harvesting and 
during transportation, handling, storage and marketing conditions that make fruits prone to fungal deterioration and decay. Filamentous fungi can enter fruits through damaged surfaces, like splits, wounds, cuts and punctures. Penicillium expansum, that produces PAT, has been reported as a major causative agent of post-harvest decay in fruits in Pakistan [21].

Table 2. A summary of studies on PAT occurrence in apple fruits and derived products.

\begin{tabular}{|c|c|c|c|c|c|}
\hline Commodity & Country & $\begin{array}{l}\text { Analytical } \\
\text { Method }\end{array}$ & Incidence (\%) & $\begin{array}{l}\text { Concentration } \\
\text { Range }(\mu \mathrm{g} / \mathrm{kg})\end{array}$ & Reference \\
\hline Apple juice for infants & Spain & MEKC $^{1}$ & 70 & LOD-29.6 & Murillo et al. [22] \\
\hline Apple juice concentrate & Spain & HPLC-UV $^{2}$ & 42.4 & LOD-74.4 & Marín et al. [23] \\
\hline Apple based food & Serbia & HPLC-UV & 43 & $3.2-30.2$ & Torović et al. [24] \\
\hline Apple juice & Sweden & HPLC-UV & 12.8 & LOD-50 & Thuvander et al. [25] \\
\hline Apple juice & Italy & HPLC-UV & 37.5 & $5.8-56.4$ & Ritieni et al. [26] \\
\hline Apple puree & Italy & HPLC-UV & 50 & $15.9-16.7$ & Ritieni et al. [26] \\
\hline Apple juice & Portugal & HPLC-DAD ${ }^{3}$ & 41 & $1.2-42$ & Barreira et al. [27] \\
\hline Apple juice & Belgium & HPLC-UV & 12 & $10.2-43.1$ & Baert et al. [28] \\
\hline Apple juice & Greece & HPLC-DAD & 100 & $0.9-36.8$ & Moukas et al. [29] \\
\hline Apple juice & Turkey & HPLC-UV & 60 & $19.1-732.8$ & Yurdun et al. [30] \\
\hline Apple juice & Tunisia & HPLC-UV & 64.3 & $4-122.4$ & Zouaoui et al. [31] \\
\hline Apple juice & Tunisia & HPLC-UV & 37 & $0-167$ & Zaied et al. [32] \\
\hline Apple juice & S. Africa & HPLC-UV & 23.5 & $5-45$ & Leggot et al. [33] \\
\hline Apple juice & S. Africa & HPLC-UV & 33.3 & $0-1650$ & Shephard et al. [34] \\
\hline Apple juice & USA & HPLC-DAD & 18.7 & LOD-467.4 & Harris et al. [35] \\
\hline Apple juice & Brazil & HPLC-DAD & 3 & 3-7 & Iha et al. [36] \\
\hline Apple fruit & Argentina & HPLC-DAD & 40.3 & LOD-19622 & Oteiza et al. [16] \\
\hline Apple juice & Malaysia & HPLC-UV & 7.7 & LOD-26.9 & Lee et al. [37] \\
\hline Apple juice & China & LC-MS 4 & 42.9 & LOD-1234.3 & Li et al. [38] \\
\hline Apple juice & Japan & LC-MS & 19.7 & $1.4-45.6$ & Ito et al. [39] \\
\hline Apple juice & S. Korea & HPLC-DAD & 12.5 & LOD-8.9 & Cho et al. [40] \\
\hline Apple juice & Iran & $\mathrm{TLC}^{5}$ & 31 & $15-285.5$ & Cheraghali et al. [41] \\
\hline Apple fruit & Pakistan & HPLC-UV & 56.4 & LOD-396 & Present study \\
\hline Apple juice & Pakistan & HPLC-UV & 62.9 & LOD-18 & Present study \\
\hline Apple juice concentrate & Pakistan & HPLC-UV & 80 & LOD-328 & Present study \\
\hline Apple puree & Pakistan & HPLC-UV & 71.4 & LOD-99 & Present study \\
\hline Apple jam & Pakistan & HPLC-UV & 30 & LOD-6 & Present study \\
\hline
\end{tabular}

${ }^{1}$ Micellar electrokinetic chromatography; ${ }^{2}$ Liquid chromatography with ultraviolet detection; ${ }^{3}$ Liquid chromatography with diode array detection; ${ }^{4}$ Liquid chromatography with mass spectrometry detection; ${ }^{5}$ Thin-layer chromatography.

The occurrence of PAT in analyzed apple varieties showed some differences that may be due to several factors, such as apple texture firmness. Previous research on PAT accumulation in numerous apple cultivars at different times during storage indicated a dependence on cultivar [42]. In Pakistan, an in vitro study conducted by Sattar et al. [43], reported that Golden Delicious (locally Shin Kulu) showed significantly higher growth of P. expansum as compared to Red Delicious (locally Tor Kulu). The authors attributed the different susceptibility to blue mold in that Golden Delicious apples have a thin cuticle and the least amount of acids and are prone to more injuries as compared to other varieties.

The factors and inducers modulating patulin synthesis in apples are still not clear, although several environmental factors and intrinsic characteristics of the fruits have been suggested to contribute to patulin accumulation [5]. Differences in susceptibility to fungal pathogens among apple cultivars might be related to the apple fruit intrinsic factors such as the content of sugars, ethylene, organic acids and phenolic compounds as well as the $\mathrm{pH}$ [44]. Among the thousands of apple cultivars, some of them show a fast response toward wounding and decaying, while other susceptible ones fail to combat pathogen attack, resulting in a large accumulation of patulin within the fruit flesh [7].

Patulin occurrence in apples is probably more affected by weather conditions before harvest and apple storage temperature than by cultivar. Higher storage temperatures will increase the probability for patulin occurrence in apple cultivars whose fruits have thin and fragile skin, but not in firm apples with thicker skin and higher flesh firmness [45]. Therefore, flesh firmness is an important sensory trait of all apple cultivars, particularly domesticated ones, that is associated with the fruit resistance to blue mold decay and patulin production [7]. In addition, the presence of a bitter pit, a physiological disorder 
of apples related to nitrogen fertilization and intensive pruning of trees, can weaken the resistance of the fruit to pathogens favoring the growth of patulin-producing fungi [45]. Likewise, phenolic compounds can have an important role as defense mechanisms against pathogens and patulin in apple cultivars [46]. All these parameters are contributing factors for the fungal deterioration and decay of apple fruits and mycotoxin production. Therefore, many factors affect the levels of contamination by patulin in apples, and it is difficult to determine which of them have been decisive in each case. However, climatic conditions before harvest (i.e., rainfall two weeks before harvest), during transportation, storage and handling, as well as retailer training are key factors that cannot be ignored. All these elements are responsible for the variation in PAT levels in the analyzed samples.

Patulin is extremely soluble in water, and highly stable at an acidic $\mathrm{pH}$, so it can migrate beyond lesion of fungal attack in stored fruits during post-harvest. Therefore, cleaning of fruits only by washing and exclusion of decayed parts cannot guarantee PAT decontamination. For instance, Olsen et al. [47] reported PAT contamination in apple jam due to surface growth of P. expansum and observed PAT penetration up to $4 \mathrm{~cm}$ layer of the jam. The proper handling and storage of fruits is the basis for the prevention of PAT [48]. In this sense, the storage temperature is a key factor to explain patulin accumulation in apples, as the higher the storage temperature, the greater the mycotoxin accumulation [5,43]. In order to increase the quality of apples in Pakistan, there is a need to establish treatment plants and cold-storage facilities for fruit preservation during off-season.

Our study indicated that patulin is frequently detected in apple and apple products throughout Pakistan and the degree of contamination is affected by multiple factors, so further studies with different cultivars in different pre- and postharvest conditions should be carried out, in order to better understand the risk for its occurrence in apple products.

\subsection{Occurrence of Patulin in Grapes Fruits and Their Products}

Incidence and contamination level of PAT in grape fruits and juices are shown in Table 3 . The results revealed that 112 out of 172 samples $(65.1 \%)$ were positive for PAT. The positivity in fresh table grapes was $59.4 \%$, while in grape juices it reached $84.6 \%$. From the eight grape varieties analyzed, the prevalence varied from $50 \%$ (White Thomson) to $66.7 \%$ (King Ruby), while the average PAT concentrations were between $33.8 \mu \mathrm{g} / \mathrm{kg}$ (White Thompson) and $90.9 \mu \mathrm{g} / \mathrm{kg}$ (Sundar Khani). Nevertheless, the differences between grapes varieties were not significant (ANOVA $p=0.9458$ ). As regards of sampling location, the incidence varied between 50\% (Lahore) and 70\% (Rawalpindi), while average concentrations ranged from $22.5 \mu \mathrm{g} / \mathrm{kg}$ (Attock) to $101.1 \mu \mathrm{g} / \mathrm{kg}$ (Rawalpindi), but differences were not significant (ANOVA $p=0.7258$ ).

PAT was also found in 33 out of 39 grape juice samples (84.6\%) from the market, at a mean concentration of $16.3 \mu \mathrm{g} / \mathrm{kg}$. The highest level of PAT was found in a sample of grapes from Faisalabad $(505 \mu \mathrm{g} / \mathrm{kg}$ ), followed by a sample of Sundar Khani grapes that amounted to $490 \mu \mathrm{g} / \mathrm{kg}$. Overall, our results highlighted that 41 samples of grapes fruits $(23.8 \%)$ surpassed the maximum regulatory limit of $50 \mu \mathrm{g} / \mathrm{kg}$, while all grapes juice samples were compliant.

Patulin has been mainly associated with apples, and apple-based products. However, the toxin may possibly contaminate the other fruits, such as grapes (Table 4). For instance, PAT was shown to be positive in 5 out of 50 samples of grapes fruits from Argentina, with a mean level of $283 \mu \mathrm{g} / \mathrm{kg}$ and a maximum value of $13,808 \mu \mathrm{g} / \mathrm{kg}$ [16]. In a previous survey carried out in Pakistan [15], high levels of patulin in grapes fruits were also determined. They found PAT in 22 out of 31 samples ( $71 \%$ positive) but average level of $504 \mu \mathrm{g} / \mathrm{kg}$ was much higher than our findings.

The presence of patulin-producing strains of Penicillium has been previously reported in grapes. Sanzani et al. [49] in Italy isolated Penicillium DNA from samples of grape must and wine contaminated with patulin (27 to $1911 \mu \mathrm{g} / \mathrm{kg}$ ). Ostry et al. [50] verified the occurrence of PAT in grape must in Czech Republic, where contamination levels varied between 119 and $644 \mu \mathrm{g} / \mathrm{kg}$. 
Table 3. Occurrence of patulin $(\mu \mathrm{g} / \mathrm{kg})$ in grapes fruits, and their derived products.

\begin{tabular}{|c|c|c|c|c|c|c|}
\hline Sample Type & Variety/Location & $\begin{array}{c}n \text { Total } \\
\text { (Positive) }\end{array}$ & $\begin{array}{c}\text { Incidence } \\
\%\end{array}$ & Mean \pm SD & Max. & $\begin{array}{c}n(\%)> \\
50 \mu \mathrm{g} / \mathrm{kg}\end{array}$ \\
\hline Grapes fruit & White Thompson ${ }^{1}$ & $6(3)$ & 50 & $33.8 \pm 50.3$ & 119 & $2(33.3)$ \\
\hline Grapes fruit & King Ruby ${ }^{1}$ & $9(6)$ & 66.7 & $52.7 \pm 60.6$ & 152 & $4(44.4)$ \\
\hline Grapes fruit & Narc Black ${ }^{1}$ & $12(7)$ & 58.3 & $60.4 \pm 93.2$ & 274 & $4(33.3)$ \\
\hline Grapes fruit & Perlette ${ }^{1}$ & $8(5)$ & 62.5 & $53.4 \pm 102.3$ & 298 & $2(25)$ \\
\hline Grapes fruit & Sundar Khani & $14(8)$ & 57.1 & $90.9 \pm 140.9$ & 490 & $6(42.9)$ \\
\hline Grapes fruit & Vitro Black & $10(6)$ & 60 & $63.6 \pm 112.9$ & 356 & $3(30)$ \\
\hline Grapes fruit & Cardinal $^{1}$ & $9(5)$ & 55.6 & $62.9 \pm 114.2$ & 310 & $2(22.2)$ \\
\hline Grapes fruit & Flame ${ }^{1}$ & $11(6)$ & 54.5 & $40.5 \pm 68.8$ & 183 & $2(18.2)$ \\
\hline Grapes fruit & Faisalabad City & $16(10)$ & 62.5 & $99.4 \pm 169.2$ & 505 & $5(31.3)$ \\
\hline Grapes fruit & Lahore City & $10(5)$ & 50 & $57.7 \pm 107.1$ & 306 & $2(20)$ \\
\hline Grapes fruit & Rawalpindi City & $10(7)$ & 70 & $101.1 \pm 139.7$ & 416 & $4(40)$ \\
\hline Grapes fruit & Attock City & $7(4)$ & 57.1 & $22.5 \pm 38.7$ & 107 & $1(14.3)$ \\
\hline Grapes fruit & Dunyapur City & $5(3)$ & 60 & $55 \pm 89.2$ & 210 & $2(40)$ \\
\hline Grapes fruit & Chakwal City & $6(4)$ & 66.7 & $44.6 \pm 70.7$ & 180 & $2(33.3)$ \\
\hline Grapes juice & Supermarket & $39(33)$ & 84.6 & $16.3 \pm 11.9$ & 39 & 0 \\
\hline Total & - & $172(112)$ & 65.1 & $53.9 \pm 98.5$ & 505 & $41(23.8)$ \\
\hline
\end{tabular}

${ }^{1}$ seedless grapes varieties.

In our study, mean PAT concentration was higher in grapes with seeds (Sundar Khani and Vitro Black) than in seedless varieties such as White Thomson. It has been reported that the absence of seeds in some grape cultivars likely results in a low content of antifungal metabolites such as tannin in fruits, making these grapes more susceptible to infection. During in vitro inoculation with Aspergillus carbonarius, progression of the rotted area of the fruit and OTA accumulation was the fastest for Thompson seedless grapes, as compared to other varieties [9]. For most grape cultivars, mold contamination was greater in rotten berries than in intact berries [51]. Therefore, keeping the berries intact is likely to be essential for the storage of table grapes.

Fungal colonization and mycotoxin production on grapes are influenced by several factors, including environmental conditions, location of the vineyard, and the characteristics of the grapes. Differences in susceptibility to fungal pathogens among grape cultivars might be related to the physical and chemical properties of grape berries. It has been found that the thickness of the berry skin, $\mathrm{pH}$, the content of reducing sugars, soluble solids and organic acids contribute to the resistance of grape berries to fungal invasion and development [52].

The fungal attack also depends on the climate circumstances, improper conditions throughout the storage, transportation and sale, resulting in decayed grapes. The grapes are highly susceptible to Penicillium species that contribute to PAT production through deterioration and decay, especially during storage [53]. It is likely that the fruit vendors did not care about the storage conditions due to a lack of knowledge and proper training required for enhancing the shelf life of fruits and their products.

In Pakistan, consumption of apple, grapes and value-added products contaminated with PAT is a matter of worry for the associated health problems. In addition, patulin is a priority pollutant in products of fruit and vegetables used for processing in global trade. Then, the presence of toxins could be a source of economic losses and failure to achieve the United Nations Sustainable Development Goals (SDGs). The findings of the present study could help to increase awareness of the problem, and to better the understanding of PAT contaminations in apple, grapes and their products among farmers, traders, end-users and regulatory authorities of Pakistan.

The occurrence of filamentous fungi in grapes may result in mycotoxins, including patulin, ochratoxin A, aflatoxins, fumonisin B2, citrinin, alternariol and tenuazonic acid, among others [54]. However, the focus of the present study was to assess the levels of PAT in fruits that are currently exported to other countries as well as consumed at gross level in Pakistan. 
Table 4. A summary of studies on patulin occurrence in grapes fruits and derived products.

\begin{tabular}{lccccc}
\hline Commodity & Country & Analytical Method & Incidence (\%) & Conc. Range ( $\mu \mathrm{g} / \mathbf{k g})$ & Reference \\
\hline Grapes fruit & Argentina & HPLC-UV & 10 & $0-13,808$ & Oteiza et al. [16] \\
Grapes juice & Austria & HPLC-UV & 45.3 & LOD-41 & SCOOP Task [55] \\
Grapes must & Austria & HPLC-UV & 52.4 & LOD-750 & SCOOP Task [55] \\
Grapes juice & Belgium & HPLC-UV & 20 & LOD-36 & SCOOP Task [55] \\
Grapes juice & Germany & HPLC-UV & 3.1 & LOD-31.5 & SCOOP Task [55] \\
Grapes must & Germany & HPLC-UV & 54 & $3.5-80$ & Majerus at al. [56] \\
Grapes juice & Germany & GC-MS & 100 & $4.9-5.2$ & Rychlik et al. [57] \\
Grapes fruit & Pakistan & HPLC-UV & 59.4 & LOD-505 & Present study \\
Grapes juice & Pakistan & HPLC-UV & 84.6 & LOD-39 & Present study \\
\hline
\end{tabular}

\section{Conclusions}

The validated analytical methodology for the determination of patulin based on HPLC with UV detection exhibited good sensibility, accuracy and precision and might be used for routine quality control and quality assurance of fruits and their products. The recognition of PAT as a hazard has risen in Pakistan over the last decade. This study provides detailed information on the risk associated with PAT in apples, grapes and their products, which poses a potential health threat to consumers due to its toxic effects. Not in vain, a significant percent of analyzed apple $(27.3 \%)$ and grape $(23.8 \%)$ samples exceeded the maximum regulatory level set at $50 \mu \mathrm{g} / \mathrm{kg}$. The findings of the current study would be useful for producers, processors and regulatory authorities to introduce policies for the prevention and control of PAT in fruits before launching to the market or exporting. We recommend regular monitoring of the incidence of PAT to maintain food safety and minimize impact on trade. Surveys on the incidence and levels of mycotoxins are of prime importance because they are reliable approaches to the current incidence of these chemical contaminants in foodstuffs. They are relevant for food safety and toxicology as the results can be compared with previous studies and make it possible to assess the effect of various factors on the occurrence of mycotoxins.

Author Contributions: Conceptualization, M.R.A.; Formal analysis, S.H.; Investigation, S.H., M.R.A., M.I. (Mazhar Iqbal), M.A., M.I. (Muhammad Imran) and A.A.; Methodology, M.I. (Mazhar Iqbal), M.A. and M.I. (Muhammad Imran); Resources, M.R.A.; Software, S.H.; Supervision, M.R.A.; Writing—original draft, S.H. and M.R.A.; Writing-review and editing, S.H., M.R.A., M.I. (Mazhar Iqbal), M.A., M.I. (Muhammad Imran) and A.A. All authors have read and agreed to the published version of the manuscript.

Funding: The current research work was financed by the Higher Education Commission of Pakistan (grant number TDF03-198) and by the Government of Aragón-Spain (grant Grupo A06-20R) and the European Regional Development Fund (FEDER 2014-2020).

Acknowledgments: The authors are extremely grateful to PAEC and NIAB-C managements to provide support, and all analytical services to carry out current $\mathrm{PhD}$ study, and whole research work in the Food Toxicology Laboratory until completion.

Conflicts of Interest: All the authors declare no conflict of interest. The above funders had performed no role in the proposal of the current study; in the collection of samples, assay analyses or elucidation of data compilation; in writing of the current manuscript, or in making decision to publish the research results.

\section{References}

1. Government of Pakistan. Fruit, Vegetables and Condiments Statistics of Pakistan 2017-2018; Government of Pakistan, Ministry of National Food Security \& Research, Economic Wing: Islamabad, Pakistan, 2019.

2. Aune, D.; Giovannucci, E.; Boffetta, P.; Fadnes, L.T.; Keum, N.; Norat, T.; Greenwood, D.C.; Riboli, E.; Vatten, L.J.; Tonstad, S. Fruit and vegetable intake and the risk of cardiovascular disease, total cancer and all-cause mortality-A systematic review and dose-response meta-analysis of prospective studies. Int. J. Epidemiol. 2017, 46, 1029-1056. [CrossRef] [PubMed]

3. Ioi, J.D.; Zhou, T.; Tsao, R.F.; Marcone, M. Mitigation of Patulin in Fresh and Processed Foods and Beverages. Toxins 2017, 9, 157. [CrossRef] [PubMed]

4. Errampalli, D. Penicillium expansum (Blue Mold). In Postharvest Decay: Control Strategies; Chapter 6; Bautista-Baños, S., Ed.; Academic Press: London, UK, 2014; pp. 189-231. 
5. Barad, S.; Sionov, E.; Prusky, D. Role of patulin in post-harvest diseases. Fungal Biol. Rev. 2016, 30, $24-32$. [CrossRef]

6. Zdzieblo, A.P.; Reuter, W.M.; Shelton, C.T. Analysis of Patulin in Apple Juice by UHPLC with UV Detection. In Perkin Elmer Application Note_Liquid Chromatography; Perkin Elmer, Inc.: Waltham, MA, USA, 2015.

7. Zhong, L.; Carere, J.; Lu, Z.; Lu, F.; Zhou, T. Patulin in Apples and Apple-Based Food Products: The Burdens and the Mitigation Strategies. Toxins 2018, 10, 475. [CrossRef]

8. Snini, S.P.; Tannous, J.; Heuillard, P.; Bailly, S.; Lippi, Y.; Zehraoui, E.; Barreau, C.; Oswald, I.P.; Puel, O. Patulin is a cultivar-dependent aggressiveness factor favouring the colonization of apples by Penicillium expansum. Mol. Plant. Pathol. 2016, 17, 920-930. [CrossRef] [PubMed]

9. Jiang, C.; Shi, J.; Zhu, C. Fruit spoilage and ochratoxin a production by Aspergillus carbonarius in the berries of different grape cultivars. Food Control 2013, 30, 93-100. [CrossRef]

10. Vidal, A.; Ouhibi, S.; Gali, R.; Hedhili, A.; De Saeger, S.; De Boevre, M. The mycotoxin patulin: An updated short review on occurrence, toxicity and analytical challenges. Food Chem. Toxicol. 2019, 129, 249-256. [CrossRef]

11. Codex Alimentarius Commission (CAC). Codex General Standard for Contaminants and Toxins in Food and Feed; Codex STAN 193-1995; CAC: Rome, Italy, 1995; p. 44. Available online: http://www.fao.org/fao-whocodexalimentarius/codex-texts/list-standards/en/ (accessed on 7 October 2020).

12. European Commission (EC). European Union Commission Regulation No. 1881/2006 setting maximum levels for certain contaminants in foodstuffs. Off. J. Eur. Union 2006, 364, 5-24.

13. AOAC International. AOAC Official Method 2000.02 Patulin in clear and cloudy apple juices and apple puree. In Official Methods of Analysis of AOAC International, 18th ed.; Horwitz, W., Ed.; AOAC International: Gaithersburg, MD, USA, 2005.

14. European Commission (EC). European Union Commission Regulation No 401/2006 laying down the methods of sampling and analysis for the official control of the levels of mycotoxins in foodstuffs. Off. J. Eur. Union 2006, 70, 12-34.

15. Iqbal, S.Z.; Malik, S.; Asi, M.R.; Selamat, J.; Malik, N. Natural occurrence of patulin in different fruits, juices and smoothies and evaluation of dietary intake in Punjab, Pakistan. Food Control 2018, 84, 370-374. [CrossRef]

16. Oteiza, J.M.; Khaneghah, A.M.; Campagnollo, F.B.; Granato, D.; Mahmoudi, M.R.; Sant'Ana, A.S.; Gianuzzi, L. Influence of production on the presence of patulin and ochratoxin A in fruit juices and wines of Argentina. LWT Food Sci. Technol. 2017, 80, 200-207. [CrossRef]

17. Vaclavikova, M.; Dzuman, Z.; Lacina, O.; Fenclova, M.; Veprikova, Z.; Zachariasova, M.; Hajslova, J. Monitoring survey of patulin in a variety of fruit-based products using a sensitive UHPLC-MS/MS analytical procedure. Food Control 2015, 47, 577-584. [CrossRef]

18. Al-Hazmi, N.A. Determination of patulin and ochratoxin A using HPLC in apple juice samples in Saudi Arabia. Saudi J. Biol. Sci. 2010, 17, 353-359. [CrossRef] [PubMed]

19. Sattar, A.; Riaz, A.; Ahmed, S.; Hassan, I. Efficacy of selected plant extracts for inhibition of Penicillium expansum growth on apple fruits. Pak. J. Phytopathol. 2014, 26, 63-66.

20. Zhang, Z.; Li, M.; Wu, C.; Peng, B. Physical adsorption of patulin by Saccharomyces cerevisiae during fermentation. J. Food Sci. Technol. 2019, 56, 2326-2331. [CrossRef]

21. Zulfiqar, S.; Tariq, F.; Perveen, K.; Ayub, N.; Mansab, S.; Batool, S.Q.; Abbasi, M.; Iram, S.; Tabassum, N. Mycotoxins analysis in fresh and dry fruits from Pakistan-A review. Am. Eurasian J. Agric. Environ. Sci. 2015, 15, 2538-2545.

22. Murillo, M.; González-Peñas, E.; Amezqueta, S. Comparison between capillary electrophoresis and high performance liquid chromatography for the study of the occurrence of patulin in apple juice intended for infants. Food Chem. Toxicol. 2010, 48, 2429-2434. [CrossRef]

23. Marín, S.; Mateo, E.M.; Sanchis, V.; Valle-Algarra, F.M.; Ramos, A.J.; Jiménez, M. Patulin contamination in fruit derivatives, including baby food, from the Spanish market. Food Chem. 2011, 124, 563-568. [CrossRef]

24. Torović, L.; Dimitrov, N.; Assunção, R.; Alvito, P. Risk assessment of patulin intake through apple-based food by infants and preschool children in Serbia. Food Addit. Contam. Part A 2017, 34, 2023-2032. [CrossRef]

25. Thuvander, A.; Moller, T.; Enghardt, H.; Jansson, A.; Salomonsson, A.-C.; Olsen, M. Dietary intake of some important mycotoxins by the Swedish population. Food Addit. Contam. 2001, 18, 696-706. [CrossRef]

26. Ritieni, A. Patulin in Italian commercial apple products. J. Agric. Food Chem. 2003, 1, 6086-6090. [CrossRef] 
27. Barreira, M.J.; Alvito, P.C.; Almeida, C.M.M. Occurrence of patulin in apple-based-foods in Portugal. Food Chem. 2010, 121, 653-658. [CrossRef]

28. Baert, K.; De Meulenaer, B.; Kamala, A.; Kasase, C.; Devlieghere, F. Occurrence of patulin in organic, conventional, and handcrafted apple juices marketed in Belgium. J. Food Prot. 2006, 69, 1371-1378. [CrossRef] [PubMed]

29. Moukas, A.; Panagiotopoulou, V.; Markaki, P. Determination of patulin in fruit juices using HPLC-DAD and GC-MSD techniques. Food Chem. 2008, 109, 860-867. [CrossRef] [PubMed]

30. Yurdun, T.; Omurtag, G.Z.; Ersoy, O. Incidence of patulin in apple juices marketed in Turkey. J. Food Prot. 2001, 64, 1851-1853. [CrossRef] [PubMed]

31. Zouaoui, N.; Sbaii, N.; Bacha, H.; Abid-Essefi, S. Occurrence of patulin in various fruit juice marketed in Tunisia. Food Control 2015, 51, 356-360. [CrossRef]

32. Zaied, C.; Abid, S.; Hlel, W.; Bacha, H. Occurrence of patulin in apple-based-foods largely consumed in Tunisia. Food Control 2013, 31, 263-267. [CrossRef]

33. Leggot, N.L.; Shephard, G.S. Patulin in South African commercial apple products. Food Control 2001, 2, 73-76. [CrossRef]

34. Shephard, G.S.; van der Westhuizen, L.; Katerere, D.R.; Herbst, M.; Pineiro, M. Preliminary exposure assessment of deoxynivalenol and patulin in South Africa. Mycotoxin Res. 2010, 26, 181-185. [CrossRef]

35. Harris, K.L.; Bobe, G.; Bourquin, L.D. Patulin surveillance in apple cider and juice marketed in Michigan. J. Food Prot. 2009, 72, 1255-1261. [CrossRef]

36. Iha, M.H.; Sabino, M. Incidence of patulin in Brazilian apple-based drinks. Food Control 2008, 19, 417-422. [CrossRef]

37. Lee, T.P.; Sakai, R.; Manaf, N.A.; Rodhi, A.M.; Saad, B. High performance liquid chromatography method for the determination of patulin and 5-hydroxymethylfurfural in fruit juices marketed in Malaysia. Food Control 2014, 38, 142-149. [CrossRef]

38. Li, X.; Li, H.; Ma, W.; Guo, Z.; Li, X.; Li, X.; Zhang, Q. Determination of patulin in apple juice by single-drop liquid-liquid-liquid microextraction coupled with liquid chromatography-mass spectrometry. Food Chem. 2018, 257, 1-6. [CrossRef] [PubMed]

39. Ito, R.; Yamazaki, H.; Inoue, K.; Yoshimura, Y.; Kawaguchi, M.; Nakazawa, H. Development of liquid chromatography-electrospray mass spectrometry for the determination of patulin in apple juice. Investigation of its contamination levels in Japan. J. Agric. Food Chem. 2004, 52, 7464-7468. [CrossRef] [PubMed]

40. Cho, M.S.; Kim, K.; Seo, E.; Kassim, N.; Mtenga, A.B.; Shim, W.B.; Lee, S.H.; Chung, D.H. Occurrence of patulin in various fruit juices from South Korea: An exposure assessment. Food Sci. Biotechnol. 2010, 19, 1-5. [CrossRef]

41. Cheraghali, A.M.; Mohammadi, H.R.; Amirahmadi, M.; Yazdanpanah, H.; Abouhossain, G.; Zamaian, F. Incidence of patulin contamination in apple juice produced in Iran. Food Control 2005, 16, 165-167. [CrossRef]

42. Salomão, B.C.; Aragao, G.M.; Churey, J.J.; Padilla-Zakour, O.I.; Worobo, R.W. Influence of storage temperature and apple variety on patulin production by Penicillium expansum. J. Food Prot. 2009, 72, 1030-1036. [CrossRef]

43. Sattar, A.; Riaz, A.; Mehmood, N.; Altaf, R.; Afzal, A.; Jabeen, Z. Effect of incubation temperature on lesion diameter of Penicillium expansum on apple fruit varieties. Int. J. Biosci. 2018, 12, 1-5.

44. Kumar, D.; Tannous, J.; Sionov, E.; Keller, N.; Prusky, D. Apple intrinsic factors modulating the global regulator, LaeA, the Patulin gene cluster and patulin accumulation during fruit colonization by Penicillium expansum. Front. Plant Sci. 2018, 9, 1094. [CrossRef]

45. Heinmaa, L.; Põldma, P.; Mirmajlessi, S.M.; Kaldmäe, H.; Vangdal, E.; Kidmose, U.; Bertelsen, M.; Lo Scalzo, R.; Fibiani, M.; Moor, U. Occurrence of mycotoxin patulin and polyphenol profile of Nordic apple juices in relation to apple cultivation system and pre-processing storage temperature. Agric. Food Sci. 2019, 28, 126-136. [CrossRef]

46. Ahmadi-Afzadi, M.; Orsel, M.; Pelletier, S.; Bruneau, M.; Proux-Wéra, E.; Nybom, H.; Renou, J.P. Genome-wide expression analysis suggests a role for jasmonates in the resistance to blue mold in apple. Plant Growth Regul. 2018, 85, 375-387. [CrossRef]

47. Olsen, M.; Lindqvist, R.; Bakeeva, A.; Su-lin, L.L.; Sulyok, M. Distribution of mycotoxins produced by Penicillium spp. inoculated in apple jam and crème fraiche during chilled storage. Int. J. Food Microbiol. 2019, 292, 13-20. [CrossRef] [PubMed]

48. Hussain, S.; Asi, M.R.; Iqbal, M.; Khalid, N.; Wajih-ul-Hassan, S.; Ariño, A. Patulin Mycotoxin in Mango and Orange Fruits, Juices, Pulps, and Jams Marketed in Pakistan. Toxins 2020, 12, 52. [CrossRef] 
49. Sanzani, S.M.; Miazzi, M.M.; Di Rienzo, V.; Fanelli, V.; Gambacorta, G.; Taurino, M.R.; Montemurro, C. A rapid assay to detect toxigenic Penicillium spp. contamination in wine and musts. Toxins 2016, 8, 235. [CrossRef]

50. Ostry, V.; Malir, F.; Cumova, M.; Kyrova, V.; Toman, J.; Grosse, Y.; Pospichalova, M.; Ruprich, J. Investigation of patulin and citrinin in grape must and wine from grapes naturally contaminated by strains of Penicillium expansum. Food Chem. Toxicol. 2018, 118, 805-811. [CrossRef] [PubMed]

51. Chunmei, J.; Junling, S.; Qi'an, H.; Yanlin, L. Occurrence of toxin-producing fungi in intact and rotten table and wine grapes and related influencing factors. Food Control 2013, 31, 5-13. [CrossRef]

52. Battilani, P.; Logrieco, A.; Giorni, P.; Cozzi, G.; Bertuzzi, T.; Pietri, A. Ochratoxin A production by Aspergillus carbonarius on some grape varieties grown in Italy. J. Sci. Food Agric. 2004, 84, 1736-1740. [CrossRef]

53. El-Samawaty, A.E.R.M.; Moslem, M.A.; Yassin, M.A.; Sayed, S.R.; El-Shikh, M.S. Control of grape blue molding Penicillia by Allium sativum. J. Pure Appl. Microbiol. 2013, 7, 1047-1053.

54. Welke, J.E. Fungal and mycotoxin problems in grape juice and wine industries. Curr. Opin. Food Sci. 2019, 29, 7-13. [CrossRef]

55. SCOOP Task 3.2.8. Assessment of Dietary Intake of Patulin by the Population of EU Member States; Majerus, P., Kapp, K., Eds.; Reports on Tasks for Scientific Cooperation, Task 3.2.8; SCOOP Report: Brussels, Belgium, 2002.

56. Majerus, P.; Hain, J.; Kölb, C. Patulin in grape must and new, still fermenting wine (Federweißer). Mycotoxin Res. 2008, 24, 135-139. [CrossRef]

57. Rychlik, M. Quantification of the mycotoxin patulin in foods. Ernährung/Nutrition 2005, 29, 9-15.

Publisher's Note: MDPI stays neutral with regard to jurisdictional claims in published maps and institutional affiliations. 